\title{
A FURTHER GENERALIZATION OF THE KAKUTANI FIXED POINT THEOREM, WITH APPLICATION TO NASH EQUILIBRIUM POINTS
}

\section{L. GLICKSBERG}

Introduction. Kakutani's fixed point theorem [3] ${ }^{1}$ states that in Euclidean $n$-space a closed point to (nonvoid) convex set map of a convex compact set into itself has a fixed point. Kakutani showed that this implied the minimax theorem for finite games. The object of this note is to point out that Kakutani's theorem may be extended to convex linear topological spaces, and implies the minimax theorem for continuous games with continuous payoff as well as the existence of Nash equilibrium points.

\section{The fixed point.}

Definition. Let $X$ be a Hausdorff linear topological space, and let $S \subset X$. A point to (nonvoid) convex set mapping

$$
\Phi: S \rightarrow X
$$

is said to be closed if the graph, $\cup_{x \in s}(x, \Phi(x))$, is closed in $X \times X$. In terms of directed systems (or nets) [1] this may be stated as either of the implications: ${ }^{2}$

$$
\text { (b) } \quad x_{\delta} \rightarrow x, y_{\delta} \in \Phi\left(x_{\delta}\right), y \text { a cluster point of }\left\{y_{\delta}\right\} \text { imply } y \in \Phi(x) \text {. }
$$

With this definition of a closed mapping we are able to extend a result of Bohnenblust and Karlin [2] to convex Hausdorff linear topologial spaces; indeed we use the exact analogue of their proof. (A linear topological space $X$ is said to be convex if there exists a base of convex neighborhoods of the zero element ( 0 ) which define the topology of $X$; we shall always take a neighborhood in such a space to be one of the convex neighborhoods, and to be symmetric: $V=-V[6])$.

Received by the editors April 24, 1951.

${ }^{1}$ Numbers in brackets refer to the references cited at the end of the paper.

2 It is obvious that (b) implies (a). To see (a) implies (b) we must resort to redirected or "sub-directed" systems. Let $y$ be a cluster point of $\left\{y_{\delta}\right\}, x_{\delta} \rightarrow x, y_{\delta} \in \Phi\left(x_{\delta}\right)$. Then let $\{U\}$ be the directed set of neighborhoods of $y$, directed by $U_{1} \leqq U_{2} \equiv U_{2} \subset U_{1}$. Set $\Delta^{\prime}=\left\{(\delta, U) \mid y_{\delta} \in U\right\},\left(\delta_{1}, U_{1}\right) \leqq\left(\delta_{2}, U_{2}\right) \equiv \delta_{1} \leqq \delta_{2} . U_{1} \leqq U_{2} . \Delta^{\prime}$ is a directed set, since if $y_{\delta_{1}} \in U_{1}, y_{\delta_{2}} \in U_{2} \exists \delta_{0} \geqq \delta_{1}, \delta_{2}$ and further a $\delta \geqq \delta_{0} \ni y_{\delta} \in U_{1} \cap U_{2}$ since $y$ is a cluster point. Now if we set $y_{(\delta, U)}=y_{\delta}$ for $(\delta, U) \in \Delta^{\prime}$, clearly $y_{(\delta, U)} \rightarrow_{\Delta^{\prime}} y$. Setting $x_{(\delta, U)}=x_{\delta}$ if $(\delta, U) \in \Delta^{\prime}$ yields $x(\delta, U) \rightarrow \Delta^{\prime} x$ clearly, and by (a) $y \in \Phi(x)$. 
Theorem. Given a closed point to convex set mapping $\Phi: S \rightarrow S$ of a convex compact subset $S$ of a convex Hausdorf linear topological space into itself there exists a fixed point $x \in \Phi(x)$.

(It is seen that this theorem duplicates the Tychonoff extension of Brouwer's theorem for Kakutani's theorem, and includes this in the same fashion that Kakutani's includes Brouwer's.)

Proof. Let $V$ be a given closed neighborhood of 0 . Since $S$ is compact there exists a $V$-dense set $\left\{x_{1}, \cdots, x_{n}\right\} \subset S$, that is, $\left\{x_{1}, \cdots, x_{n}\right\} \ni$

$$
S \subset \bigcup_{i=1}^{n}\left(x_{i}+V\right)
$$

Let $S_{V}$ be the convex hull of $\left\{x_{1}, \cdots, x_{n}\right\}$ and let

$$
\Phi_{V}(x)=(\Phi(x)+V) \cap S_{V} .
$$

Now $\Phi_{V}$ is of course a point to convex set mapping ${ }^{3}$ which in particular takes $S_{V} \rightarrow S_{V}$. Moreover $\Phi_{V}$ is a closed mapping. For, if $x_{\delta} \rightarrow x, y_{\delta} \in \Phi_{V}\left(x_{\delta}\right)$, and $y_{\delta} \rightarrow y$ then

$$
y_{\delta} \in\left(\Phi\left(x_{\delta}\right)+V\right) \cap S_{V} .
$$

Hence there exist $z_{\delta} \in \Phi\left(x_{\delta}\right)$ and $v_{\delta} \in V$ such that $y_{\delta}=z_{\delta}+v_{\delta} \in S_{V}$. Since $z_{\delta} \in \Phi\left(x_{\delta}\right) \subset S$ and since $S$ is compact, $\left\{z_{\delta}\right\}$ has a cluster point $z$ in $S$. $z$ belongs to $\Phi(x)$ since $x_{8} \rightarrow x$ and $\Phi$ is a closed mapping. Since $v_{8}=y_{8}$ $-z_{\delta}$ and $y_{\delta} \rightarrow y,\left\{v_{\delta}\right\}$ must have $v=y-z$ as a cluster point. $v$ belongs to $V$ since $v_{8} \in V$ and $V$ is closed. Thus $y=z+v \in \Phi(x)+V$. On the other hand $y \in S_{V}$ since $y_{\delta} \in \Phi_{V}\left(x_{\delta}\right) \subset S_{V}, y_{\delta} \rightarrow y$ and $S_{V}$ is closed. Thus we have

$$
y \in(\Phi(x)+V) \cap S_{V}=\Phi_{V}(x) .
$$

Hence by the Kakutani fixed point theorem for Euclidean spaces (since the relative topology on $S_{V}$ is Euclidean), we have $x_{V} \in S_{V}$, $x_{V} \in \Phi_{V}\left(x_{V}\right)$.

Now the closed neighborhoods of 0 form a natural directed set under

$$
V_{1} \leqq V_{2} \equiv V_{2} \subset V_{1}
$$

so that $\left\{x_{V}\right\}$ is a directed system. Hence we have a cluster point $x$ of $\left\{x_{V}\right\}$. Using the process indicated in footnote 2 , we form

$$
\Delta^{\prime}=\left\{(V, U) \mid x_{\nabla} \in U, U \text { a neighborhood of } x\right\}
$$

${ }^{3} \Phi_{V}(x)$ is never void since there exists an $x_{i} \in \Phi(x)+V$ whence $x_{i} \in(\Phi(x)+V)$ $\cap S_{V}=\Phi_{V}(x)$. 
which is a directed set, and set

$$
x_{(V, U)}=x_{V} \text { for }(V, U) \in \Delta^{\prime},
$$

so that

$$
x_{(\nabla, U)} \underset{\Delta^{\prime}}{\longrightarrow} x,
$$$$
\text { for any } V_{0} \text { there exist } U \text { and } V \geqq V_{0} \ni(V, U) \in \Delta^{\prime} \text {. }
$$

Then we have $z_{(V, U)}$ such that

$$
x_{(V, U)}-z_{(V, U)} \in V \quad \text { and } \quad z_{(V, U)} \in \Phi\left(x_{(V, U)}\right)
$$

from $x_{V} \in \Phi\left(x_{V}\right)+V$, so that $z_{(V, U)} \rightarrow_{\Delta^{\prime}} x$ by (2). But then $x \in \Phi(x)$ since $\Phi$ is closed, and the theorem is proved. ${ }^{4}$

2. Nash equilibrium points. Consider an $n$-person game played over $n$ compact Hausdorff pure strategy spaces $A_{1}, \cdots, A_{n}$, in which the payoff to player $i$ is

$$
M_{i}\left(x_{1}, \cdots, x_{n}\right),
$$

a real-valued, continuous function over $A_{1} \times A_{2} \times \cdots \times A_{n}$. According to Nash [4], a set of mixed strategies $\left(f_{1}^{0}, \cdots, f_{n}^{0}\right)$ (which we interpret here as regular measures such that $f_{i}\left(A_{i}\right)=1$ ) is an equilibrium point of the game if, for each $i$,

$$
\int d f_{1}^{0} \int d f_{2}^{0} \cdots \int M_{i} d f_{n}^{0}
$$

is the maximum of all similar expressions in which $f_{i}^{0}$ is replaced by an arbitrary strategy $f_{i}$. By means of the preceding theorem we shall show that equilibrium points exist; we treat for convenience the case $n=2$, the extension to the general case being obvious.

Let the players be I and II, the payoff to I be $M(x, y)$, to II be $N(x, y)$, both continuous on the compact Hausdorff space $A_{\mathrm{I}} \times A_{\mathrm{II}}$. Let $S_{\mathrm{I}}$ and $S_{\mathrm{II}}$ be the sets of mixed strategies for I and II. These are subsets of the spaces of functionals on the Banach spaces of continuous functions $C\left(A_{\mathrm{I}}\right), C\left(A_{\mathrm{II}}\right)$, on $A_{\mathrm{I}}$ and $A_{\mathrm{II}}$, and are $\omega^{*}$ compact. Since the $\omega^{*}$ topology is convex, that is, the space $C\left(A_{\mathrm{I}}\right)^{*}$ (under the $\omega^{*}$ topology) is a convex Hausdorff linear topological space, we may apply Theorem 1 to mappings on $S_{\mathrm{I}}$ or $S_{\mathrm{II}}$ or indeed

4 When $\Phi$ is a point-to-point mapping (Tychonoff's case), the approximation of $\Phi$ by a finite-dimensional point-to-convex set mapping $\Phi_{\boldsymbol{V}}$ seems simpler and more direct than the usual approximation by a finite-dimensional point-to-point mapping which involves linear interpolation. The technique is due to Bohnenblust and Karlin. 
to $S_{\mathrm{I}} \times S_{\mathrm{II}} \subset C\left(A_{\mathrm{I}}\right)^{*} \times C\left(A_{\mathrm{II}}\right)^{*}$.

Set

$$
E(f, g)=\iint M d f(x) d g(y), \quad F(f, g)=\iint N d f(x) d g(y)
$$

which are, respectively, the expectations of I and II if I uses $f$, II uses $g$. We seek a pair $\left(f^{0}, g^{0}\right)$ such that

$$
E\left(f^{0}, g^{0}\right)=\sup _{f} E\left(f, g^{0}\right), \quad F\left(f^{0}, g^{0}\right)=\sup _{\sigma} F\left(f^{0}, g\right) .
$$

For every $f$, set

$$
G(f)=\left\{g \mid\left(F(f, g)=\sup _{g^{\prime}} F\left(f, g^{\prime}\right)\right)\right\}
$$

and for every $g$ set

$$
\mathcal{f}(g)=\left\{f \mid\left(E(f, g)=\sup _{\boldsymbol{f}^{\prime}} E\left(f^{\prime}, g\right)\right)\right\} .
$$

Both $\mathcal{F}(g)$ and $G(f)$ are evidently convex, and are easily seen to be nonvoid and $\omega^{*}$ closed, hence the mapping

$$
(f, g) \rightarrow(\mathcal{F}(g), G(f))
$$

is a point to convex set mapping of $S_{\mathrm{I}} \times S_{\mathrm{II}} \rightarrow S_{\mathrm{I}} \times S_{\mathrm{II}}$. Moreover it is easily seen that the mapping is closed since $E$ and $F$ are continuous functions on $S_{\mathrm{I}} \times S_{\mathrm{II}}$. Hence by the fixed point theorem there exists a pair $\left(f^{0}, g^{0}\right)$ such that

$$
\left(f^{0}, g^{0}\right) \in\left(\mathcal{F}\left(g^{0}\right), G\left(f^{0}\right)\right)
$$

or

$$
E\left(f^{0}, g^{0}\right)=\sup _{f} E\left(f, g^{0}\right), \quad F\left(f^{0}, g^{0}\right)=\sup _{\bullet} F\left(f^{0}, g\right) .
$$

Thus equilibrium points exist. An obvious consequence is the existence of such points in the game in which only a convex $\omega^{*}$ closed subset of $S_{i}$ may be played. Another obvious consequence is the minimax theorem for the two person zero sum continuous game, wherein $N=-M$, for then

$$
\begin{aligned}
F(f, g) & =-\iint M d f(x) d g(y), \\
F\left(f^{0}, g^{0}\right) & =-\inf _{0} \iint M d f^{0}(x) d g(y),
\end{aligned}
$$




$$
E\left(f^{0}, g^{0}\right)=\sup _{f} \iint M d f(x) d g^{0}(y)
$$

so that

$$
\iint M d f^{0}(x) d g(y) \geqq \iint M d f^{0}(x) d g^{0}(y) \geqq \iint M d f(x) d g^{0}(y)
$$

whence the minimax relation follows, and $f^{0}$ and $g^{0}$ appear as optimal strategies [5].

\section{REFERENCES}

1. G. Birkhoff, Lattice theory, Amer. Math. Soc. Colloquium Publications, vol. 25.

2. H. F. Bohnenblust and S. Karlin, On a theorem of Ville, Contributions to the theory of games, Annals of Mathematics Studies, No. 24.

3. S. Kakutani, $A$ generalization of Brouwer's fixed point theorem, Duke Math. J. vol. 7 (1941) pp. 457-459.

4. J. F. Nash, Jr., Equilibrium points in N-person games, Proc. Nat. Acad. Sci. U.S.A. vol. 36 (1950) pp. 48-49.

5. J. von Neumann and $\mathrm{O}$. Morgenstern, Theory of games and economic behavior, Princeton University Press, 1947.

6. J. V. Wehausen, Transformations in linear topological spaces, Duke Math. J. vol. 4 (1938) pp. 157-169.

7. A. Tychonoff, Ein Fixpunktsatz, Math. Ann. vol. 111 (1935) pp. 767-776.

The Rand Corporation 